\title{
Quantum Sufficiency in the Operator Algebra Framework
}

\author{
Andrzej Łuczak
}

Received: 8 February 2013 / Accepted: 15 July 2013 / Published online: 1 August 2013

(C) The Author(s) 2013. This article is published with open access at Springerlink.com

\begin{abstract}
The paper is devoted to the investigation of the notion of sufficiency in quantum statistics. Three kinds of this notion are considered: plain sufficiency (called simply: sufficiency), Petz's sufficiency, and Umegaki's sufficiency. The problem of the existence and structure of the minimal sufficient subalgebra is analyzed in some detail, conditions yielding equivalence of the three modes of sufficiency are considered, and quantum Basu's theorem is obtained. Moreover, it is shown that an interesting "factorization theorem" of Jenčová and Petz needs some corrections to hold true.
\end{abstract}

Keywords Quantum sufficiency · Von Neumann algebra · Conditional expectation · Normal states

\section{Introduction}

Let $\mathfrak{M}$ be a von Neumann algebra, let $\mathfrak{N}$ be its von Neumann subalgebra, and let $\left\{\rho_{\theta}\right.$ : $\theta \in \Theta\}$ be a family of normal states on $\mathfrak{M}$. The most general notion of sufficiency of the subalgebra $\mathfrak{N}$ for the family $\left\{\rho_{\theta}: \theta \in \Theta\right\}$ was introduced by Petz in $[5,6]$ as a generalization of sufficiency in Umegaki's sense considered earlier in $[8,9]$. It was further investigated in $[1,2]$. In this setup the sufficiency of $\mathfrak{N}$ means the existence of a two-positive map $\alpha: \mathfrak{M} \rightarrow$ $\mathfrak{N}$ such that

$$
\rho_{\theta} \circ \alpha=\rho_{\theta}, \quad \theta \in \Theta .
$$

(Note that if the map $\alpha$ is a conditional expectation then we get sufficiency in Umegaki's sense.) However, it seems equally interesting to investigate a natural generalization of this notion which would consist in giving up the, rather technical, requirement of two-positivity

Work supported by NCN grant no 2011/01/B/ST1/03994.

A. Łuczak ( ()

Faculty of Mathematics and Computer Science, Łódź University, ul. S. Banacha 22, 90-238 Łódź,

Poland

e-mail: anluczak@math.uni.lodz.pl 
and replacing it by mere positivity. This approach is additionally motivated by considerations from quantum hypothesis testing theory. To briefly explain this standpoint assume that we are given a finite number of states $\left\{\rho_{1}, \ldots, \rho_{n}\right\}$ which can occur with a priori probabilities $\left(\pi_{1}, \ldots, \pi_{n}\right)$, respectively, and we seek a measurement $M=\left(M_{1}, \ldots, M_{n}\right)$ which minimizes the so-called Bayes risk

$$
r(M, \pi)=\sum_{i, j=1}^{n} \pi_{i} L(i, j) \rho_{i}\left(M_{j}\right),
$$

where $L(i, j)$ are real numbers (the function $(i, j) \mapsto L(i, j)$ is called a loss function), and measurement $M=\left(M_{1}, \ldots, M_{n}\right)$ means positive operators $M_{j} \in \mathfrak{M}$ such that $\sum_{j=1}^{n} M_{j}=\mathbb{1}$. Now it is clear that an appropriate notion of sufficiency for this problem would consist in the existence of a positive unital map $\alpha: \mathfrak{M} \rightarrow \mathfrak{N}$ into a von Neumann subalgebra $\mathfrak{N}$ of $\mathfrak{M}$ such that $\rho_{i} \circ \alpha=\rho_{i}$ for all $i$, since then $N=\left(N_{1}, \ldots, N_{n}\right)$ defined as $N_{j}=\alpha\left(M_{j}\right)$ is a measurement in $\mathfrak{N}$ such that $r(N, \pi)=r(M, \pi)$, so an optimal measurement can be found in the subalgebra $\mathfrak{N}$. Exactly the same argument applies if one considers the minimax risk instead of the Bayes one.

The investigation of this general form of sufficiency is the purpose of the paper. In particular, we examine various questions concerning the notion of minimality, show that under the additional assumption of completeness all the three notions of sufficiency: the one considered in the paper, Petz's sufficiency and Umegaki's sufficiency coincide, and obtain a quantum version of Basu's theorem. We also comment on a "factorization theorem" by Jenčová and Petz showing by means of examples that in its present form it does not hold and needs some corrections.

It is worth noting that the analysis of minimality in the first part of the paper can be adapted to Petz's definition of sufficiency yielding a new description of the minimal sufficient subalgebra.

\section{Preliminaries and Notation}

Let $\mathfrak{M}$ be an arbitrary von Neumann algebra with identity $\mathbb{1}$.

A state on $\mathfrak{M}$ is a bounded positive linear functional $\rho: \mathfrak{M} \rightarrow \mathbb{C}$ of norm one. A state is called normal if it is continuous in the $\sigma$-weak topology on $\mathfrak{M}$. For a normal state $\rho$ its support, denoted by $\mathrm{s}(\rho)$, is defined as the smallest projection in $\mathfrak{M}$ such that $\rho(\mathrm{s}(\rho))=$ $\rho(\mathbb{1})$. We have

$$
\rho(\mathrm{s}(\rho) A)=\rho(A \mathrm{~s}(\rho))=\rho(A), \quad A \in \mathfrak{M},
$$

and if $\rho(\mathrm{s}(\rho) A \mathrm{~s}(\rho))=0$ for $\mathrm{s}(\rho) A \mathrm{~s}(\rho) \geq 0$ then $\mathrm{s}(\rho) A \mathrm{~s}(\rho)=0$.

Let $\left\{\rho_{\theta}: \theta \in \Theta\right\}$ be a family of normal states on a von Neumann algebra $\mathfrak{M}$. This family is said to be faithful if for each positive element $A \in \mathfrak{M}$ from the equality $\rho_{\theta}(A)=0$ for all $\theta \in \Theta$ it follows that $A=0$. It is seen that the faithfulness of the family is equivalent to the relation

$$
\bigvee_{\theta \in \Theta} \mathrm{s}\left(\rho_{\theta}\right)=\mathbb{1} .
$$

Let $P$ be a projection in a von Neumann algebra $\mathfrak{M}$. A reduced von Neumann algebra $\mathfrak{M}_{P}$ is defined as

$$
\mathfrak{M}_{P}=\{P A P \mid P(\mathcal{H}): A \in \mathfrak{M}\}
$$


Let $\mathfrak{M}$ and $\mathfrak{N}$ be von Neumann algebras. A linear map $\alpha: \mathfrak{M} \rightarrow \mathfrak{N}$ is called normal if it is continuous in the $\sigma$-weak topologies on $\mathfrak{M}$ and $\mathfrak{N}$, respectively. It is called unital if $\alpha(\mathbb{1})=\mathbb{1}$

For an arbitrary subset $\mathfrak{A}$ of $\mathbb{B}(\mathcal{H})$ by $W^{*}(\mathfrak{A})$ we shall denote the von Neumann algebra generated by $\mathfrak{A}$, i.e. the smallest von Neumann algebra containing $\mathfrak{A}$.

Let $\mathfrak{M}$ be a von Neumann algebra, let $\left\{\rho_{\theta}: \theta \in \Theta\right\}$ be a family of normal states on $\mathfrak{M}$, and let $\mathfrak{N}$ be a von Neumann subalgebra of $\mathfrak{M}$. $\mathfrak{N}$ is said to be sufficient for the family of states $\left\{\rho_{\theta}: \theta \in \Theta\right\}$ if there exists a linear positive normal unital map $\alpha: \mathfrak{M} \rightarrow \mathfrak{N}$ such that

$$
\rho_{\theta} \circ \alpha=\rho_{\theta}, \quad \text { for all } \theta \in \Theta \text {. }
$$

If the map $\alpha$ above is two-positive then $\mathfrak{N}$ is said to be sufficient in Petz's sense, and if it is a conditional expectation onto $\mathfrak{N}$ then $\mathfrak{N}$ is said to be sufficient in Umegaki's sense. If the algebra $\mathfrak{N}$ is sufficient and contained in any other sufficient (sufficient in Petz's or Umegaki's sense, respectively) algebra then $\mathfrak{N}$ is said to be minimal. It is clear that a minimal sufficient (in any sense) subalgebra is unique (if it exists).

\section{Minimal Sufficient Subalgebras}

The existence of sufficient subalgebras of a von Neumann algebra $\mathfrak{M}$ is obvious because $\mathfrak{M}$ itself is certainly sufficient. However, with minimality the question is more subtle.

Theorem 1 Let $\left\{\rho_{\theta}: \theta \in \Theta\right\}$ be a faithful family of normal states on a von Neumann algebra $\mathfrak{M}$. There exists the minimal sufficient subalgebra of the algebra $\mathfrak{M}$.

Proof Let $\mathcal{S}$ be the family of all normal positive unital maps on $\mathfrak{M}$ such that the states $\rho_{\theta}$ are invariant with respect to the maps from $\mathcal{S}$. It is seen that $\mathcal{S}$ is a non-empty (because it contains the identity map) semigroup. Let $\mathfrak{A}$ be the set of the fixed points of the maps from $\mathcal{S}$, i.e.

$$
\mathfrak{A}=\{A \in \mathfrak{M}: \alpha(A)=A \text { for all } \alpha \in \mathcal{S}\} .
$$

From the ergodic theorem for von Neumann algebras (see [7]) it follows that $\mathfrak{A}$ is a $J W^{*}$ algebra, i.e. a $\sigma$-weakly closed linear subspace of $\mathbb{B}(\mathcal{H})$ containing the unit $\mathbb{1}$, closed with respect to the *-operation and the Jordan product $A \circ B=(A B+B A) / 2$, and there exists a positive normal unital projection $\mathbb{E}$ from $\mathfrak{M}$ onto $\mathfrak{A}$ such that

$$
\rho_{\theta} \circ \mathbb{E}=\rho_{\theta} \quad \text { for all } \theta \in \Theta,
$$

and

$$
\mathbb{E}(A \circ B)=A \circ \mathbb{E}(B), \quad \text { for all } A \in \mathfrak{A}, B \in \mathfrak{M} .
$$

Denote by $\mathfrak{M}_{\min }$ the von Neumann algebra generated by $\mathfrak{A}, \mathfrak{M}_{\min }=W^{*}(\mathfrak{A})$. Clearly, $\mathfrak{M}_{\min }$ is sufficient.

Let $\mathfrak{N}$ be an arbitrary sufficient subalgebra of the algebra $\mathfrak{M}$, and let $\alpha: \mathfrak{M} \rightarrow \mathfrak{N}$ be a map defining this sufficiency. Then $\alpha \in \mathcal{S}$, and for arbitrary $A \in \mathfrak{A}$ we have $A=\alpha(A) \in \mathfrak{N}$, which shows that $\mathfrak{A} \subset \mathfrak{N}$, consequently, $\mathfrak{M}_{\min } \subset \mathfrak{N}$ and thus $\mathfrak{M}_{\text {min }}$ is minimal. 
Remark 1 If in the proof of the above theorem we defined $\mathcal{S}$ as the family of all normal two-positive unital maps on $\mathfrak{M}$ such that the states $\rho_{\theta}$ are $\mathcal{S}$-invariant, then from the ergodic theorem for von Neumann algebras in [3], it would follow that $\mathfrak{A}$ is a von Neumann algebra and the map $\mathbb{E}$ is a conditional expectation. Consequently, $\mathfrak{A}$ would be minimal sufficient in Umegaki's sense. Thus Theorem 1 in this setup would give the existence and a description of the minimal sufficient in Umegaki's sense subalgebra of $\mathfrak{M}$. It is interesting to compare the above description of minimality with the one given in [1] where the minimal sufficient in Umegaki's sense subalgebra of $\mathfrak{M}$ is characterized as the von Neumann algebra generated by the Connes' cocycles $\left[D \rho_{\theta}: D \omega\right]_{t}, t \in \mathbb{R}, \theta \in \Theta$, where $\omega$ is a faithful normal state on $\mathfrak{M}$ expressed as a convex combination of some $\rho_{\theta}$.

Also, it is worth noticing how using ergodic theory for von Neumann algebras gives a simple proof of the existence of minimal sufficient subalgebra in any sense, especially when compared with an involved proof of this fact only for Petz's sufficiency obtained with the help of the Connes' cocycles (see [1, 2, 4]).

The assumption of the faithfulness of the states in question is essential as the following example shows.

Example 1 Let $\mathfrak{M}=\mathbb{B}\left(\mathbb{C}^{3}\right)$, and let $\psi_{1}, \psi_{2}$ be vectors of the standard basis in $\mathbb{C}^{3}$

$$
\psi_{1}=\left(\begin{array}{l}
1 \\
0 \\
0
\end{array}\right), \quad \psi_{2}=\left(\begin{array}{l}
0 \\
1 \\
0
\end{array}\right)
$$

Define the states $\rho_{1}$ and $\rho_{2}$ as

$$
\rho_{1}(A)=\left\langle\psi_{1} \mid A \psi_{1}\right\rangle, \quad \rho_{2}(A)=\left\langle\psi_{2} \mid A \psi_{2}\right\rangle, \quad A \in \mathfrak{M},
$$

and consider von Neumann algebras

$$
\mathfrak{M}_{1}=\left\{\left[\begin{array}{lll}
a & 0 & 0 \\
0 & b & 0 \\
0 & 0 & b
\end{array}\right]: a, b \in \mathbb{C}\right\}, \quad \mathfrak{M}_{2}=\left\{\left[\begin{array}{lll}
a & 0 & 0 \\
0 & b & 0 \\
0 & 0 & a
\end{array}\right]: a, b \in \mathbb{C}\right\}
$$

Define maps $\mathbb{E}_{i}: \mathfrak{M} \rightarrow \mathfrak{M}_{i}, i=1,2$, by the formulas

$$
\begin{aligned}
& \mathbb{E}_{1}\left(\left[\begin{array}{lll}
a_{11} & a_{12} & a_{13} \\
a_{21} & a_{22} & a_{23} \\
a_{31} & a_{32} & a_{33}
\end{array}\right]\right)=\left[\begin{array}{ccc}
a_{11} & 0 & 0 \\
0 & a_{22} & 0 \\
0 & 0 & a_{22}
\end{array}\right] \\
& \mathbb{E}_{2}\left(\left[\begin{array}{lll}
a_{11} & a_{12} & a_{13} \\
a_{21} & a_{22} & a_{23} \\
a_{31} & a_{32} & a_{33}
\end{array}\right]\right)=\left[\begin{array}{ccc}
a_{11} & 0 & 0 \\
0 & a_{22} & 0 \\
0 & 0 & a_{11}
\end{array}\right] .
\end{aligned}
$$

It is easily verified that $\mathbb{E}_{i}$ is a conditional expectation onto $\mathfrak{M}_{i}$ such that the states $\rho_{1}$ and $\rho_{2}$ are $\mathbb{E}_{i}$-invariant, thus the algebras $\mathfrak{M}_{1}$ and $\mathfrak{M}_{2}$ are sufficient in Umegaki's sense for the family $\left\{\rho_{1}, \rho_{2}\right\}$. Suppose that there exists the minimal sufficient subalgebra $\mathfrak{M}_{\min }$. Then

$$
\mathfrak{M}_{\min } \subset \mathfrak{M}_{1} \cap \mathfrak{M}_{2}=\mathbb{C} \mathbb{1}
$$


so $\mathfrak{M}_{\text {min }}=\mathbb{C} \mathbb{1}$, which is impossible since the algebra $\mathbb{C} \mathbb{1}$ is sufficient only for one (arbitrary) state, namely, if $\rho$ is any state then the map $\alpha$ defining sufficiency must be of the form

$$
\alpha(A)=\rho(A) \mathbb{1} .
$$

Now drop the assumption of the faithfulness of the family $\left\{\rho_{\theta}: \theta \in \Theta\right\}$. Put

$$
P=\bigvee_{\theta \in \Theta} \mathrm{s}\left(\rho_{\theta}\right)
$$

and consider the reduced algebra $\mathfrak{M}_{P}$. Denote its elements by $A_{P}$, thus

$$
A_{P}=P A P \mid P(\mathcal{H}),
$$

and

$$
\mathfrak{M}_{P}=\left\{A_{P}: A \in \mathfrak{M}\right\} .
$$

Define a "restriction" of the states $\rho_{\theta}$ to the algebra $\mathfrak{M}_{P}$ by the formula

$$
\rho_{\theta}^{P}\left(A_{P}\right)=\rho_{\theta}(P A P)=\rho_{\theta}(A) .
$$

Observe that they are well defined, since if

$$
P A_{1} P\left|P(\mathcal{H})=P A_{2} P\right| P(\mathcal{H}),
$$

then obviously

$$
P A_{1} P=P A_{2} P \text {. }
$$

The family $\left\{\rho_{\theta}^{P}: \theta \in \Theta\right\}$ is faithful, so according to Theorem 1 there exists the minimal sufficient subalgebra $\mathfrak{M}_{P}^{0}$ of $\mathfrak{M}_{P}$ for this family. The algebra $\mathfrak{M}_{P}^{0}$ can be considered "minimal" in the sense described in the following theorem.

Theorem 2 Let $\left\{\rho_{\theta}: \theta \in \Theta\right\}$ be an arbitrary family of normal states on a von Neumann algebra $\mathfrak{M}$. For each sufficient for this family von Neumann subalgebra $\mathfrak{N}$ of $\mathfrak{M}$ we have $\mathfrak{M}_{P}^{0} \subset \mathfrak{N}_{P}$, where by a slight abuse of notation ( $P$ need not belong to $\mathfrak{N}$ ) we set $\mathfrak{N}_{P}=$ $\{P A P \mid P(\mathcal{H}): A \in \mathfrak{N}\}$. (Warning: $\mathfrak{N}_{P}$ need not be an algebra.)

Proof Let $\mathfrak{N}$ be a sufficient subalgebra, and let $\alpha: \mathfrak{M} \rightarrow \mathfrak{N}$ be a map defining this sufficiency. Define a map $\alpha_{P}: \mathfrak{M}_{P} \rightarrow \mathfrak{N}_{P} \subset \mathfrak{M}_{P}$ by the formula

$$
\alpha_{P}\left(A_{P}\right)=(\alpha(P A P))_{P}=P \alpha(P A P) P \mid P(\mathcal{H}) .
$$

This map is clearly linear normal and positive. For the map $\alpha$ we have

$$
\rho_{\theta}\left(P \alpha\left(P^{\perp}\right) P\right)=0, \quad \text { for all } \theta \in \Theta,
$$

and since $P \alpha\left(P^{\perp}\right) P \geq 0$ we get

$$
P \alpha\left(P^{\perp}\right) P=0 .
$$

From this equality we obtain

$$
P \alpha(P) P=P,
$$


which implies

$$
\alpha_{P}\left(\mathbb{1}_{P(\mathcal{H})}\right)=P \alpha(P) P|P(\mathcal{H})=P| P(\mathcal{H})=\mathbb{1}_{P(\mathcal{H})},
$$

so $\alpha_{P}$ is unital. Further, for each $\theta \in \Theta$ we have

$$
\rho_{\theta}^{P}\left(\alpha_{P}\left(A_{P}\right)\right)=\rho_{\theta}(P \alpha(P A P) P)=\rho_{\theta}(P A P)=\rho_{\theta}^{P}\left(A_{P}\right),
$$

showing that the states $\rho_{\theta}^{P}$ are $\alpha_{P}$-invariant. Take arbitrary $A_{P} \in \mathfrak{M}_{P}^{0}$. From the description of the minimal sufficient subalgebra obtained in the proof of Theorem 1 it follows that $\alpha_{P}\left(A_{P}\right)=A_{P}$, consequently, $A_{P} \in \mathfrak{N}_{P}$, thus $\mathfrak{M}_{P}^{0} \subset \mathfrak{N}_{P}$.

The next two examples bear a direct connection to the factorization theorem in [1] ([1, Theorem 4]). For this reason we shall use the notation employed there.

Example 2 Let $\mathfrak{M}=\mathbb{B}(\mathcal{H})$, with $\operatorname{dim} \mathcal{H}=\infty$, and let

$$
\sum_{i=1}^{\infty} P_{i}=\mathbb{1}
$$

be a countable partition of the identity such that the $P_{i}$ are finite-dimensional projections. Denote

$$
\mathcal{H}_{i}=P_{i}(\mathcal{H}), \quad d_{i}=\operatorname{dim} \mathcal{H}_{i} .
$$

Let $\left\{\rho_{i}: i=1,2, \ldots\right\}$ be normal states such that $\mathrm{s}\left(\rho_{i}\right)=P_{i}$, and denote by $D_{i}$ their density matrices, i.e.

$$
\rho_{i}(A)=\operatorname{tr} D_{i} A, \quad A \in \mathbb{B}(\mathcal{H}) .
$$

The family $\left\{\rho_{i}: i=1,2, \ldots\right\}$ is faithful and from the condition on the support we obtain $D_{i} \leq P_{i}$. Denote by $c_{k}^{(i)}, k=1, \ldots, d_{i}$, the eigenvalues of $D_{i}$, and let

$$
S\left(\rho_{i}\right)=-\sum_{k=1}^{d_{i}} c_{k}^{(i)} \log c_{k}^{(i)} \quad\left(\log \equiv \log _{2}\right)
$$

be the entropy of the state $\rho_{i}$. Choose our states $\rho_{i}$ in such a way that $S\left(\rho_{i}\right) \leq c$ for all $i$, where $c$ is a given constant.

Put

$$
\omega=\sum_{i=1}^{\infty} \frac{1}{2^{i}} \rho_{i} .
$$

The density matrix of $\omega$ has the form

$$
D_{\omega}=\sum_{i=1}^{\infty} \frac{1}{2^{i}} D_{i}
$$

so for the entropy of $\omega$ we get

$$
S(\omega)=-\sum_{i=1}^{\infty} \sum_{k=1}^{d_{i}} \frac{c_{k}^{(i)}}{2^{i}} \log \frac{c_{k}^{(i)}}{2^{i}}=-\sum_{i=1}^{\infty} \frac{1}{2^{i}} \sum_{k=1}^{d_{i}} c_{k}^{(i)}\left[\log c_{k}^{(i)}-\log 2^{i}\right]
$$




$$
\begin{aligned}
& =-\sum_{i=1}^{\infty} \frac{1}{2^{i}}\left[\sum_{k=1}^{d_{i}} c_{k}^{(i)} \log c_{k}^{(i)}-\sum_{k=1}^{d_{i}} i c_{k}^{(i)}\right]=\sum_{i=1}^{\infty} \frac{1}{2^{i}}\left[S\left(\rho_{i}\right)+i\right] \\
& \leq c+\sum_{i=1}^{\infty} \frac{i}{2^{i}}<\infty .
\end{aligned}
$$

The pair (M, $\left.\left\{\rho_{i}: i=1,2, \ldots\right\}\right)$ constitutes what in [1] is called a statistical experiment dominated by a faithful normal state $\omega$. Let $\mathfrak{M}_{0}$ be a von Neumann subalgebra of $\mathfrak{M}$ defined as

$$
\mathfrak{M}_{0}=\left\{\sum_{i=1}^{\infty} a_{i} P_{i}: a_{i} \in \mathbb{C}, \sup _{1 \leq i<\infty}\left|a_{i}\right|<\infty\right\} .
$$

Define a map $\mathbb{E}: \mathfrak{M} \rightarrow \mathfrak{M}_{0}$ by the formula

$$
\mathbb{E}(A)=\sum_{i=1}^{\infty} \rho_{i}(A) P_{i}, \quad A \in \mathfrak{M} .
$$

It is easily seen that $\mathbb{E}$ is a normal conditional expectation such that the states $\rho_{i}$ are $\mathbb{E}$-invariant, so $\mathfrak{M}_{0}$ is sufficient for $\left\{\rho_{i}: i=1,2, \ldots\right\}$ in Umegaki's sense. Clearly, $\omega$ is also $\mathbb{E}$-invariant, consequently, $\mathfrak{M}_{0}$ is invariant with respect to the modular automorphism group $\left\{\sigma_{t}^{\omega}\right\}$. For each $B=\sum_{i=1}^{\infty} a_{i} P_{i} \in \mathfrak{M}_{0}$ we have

$$
\rho_{j}(B)=\sum_{i=1}^{\infty} a_{i} \rho_{j}\left(P_{i}\right)=a_{j}=\operatorname{tr}\left(\frac{1}{d_{j}} P_{j}\right)\left(\sum_{i=1}^{\infty} a_{i} P_{i}\right)=\operatorname{tr}\left(\frac{1}{d_{j}} P_{j}\right) B,
$$

which means that the density matrix of the restricted state $\rho_{j} \mid \mathfrak{M}_{0}$ is

$$
D_{j, 0}=\frac{1}{d_{j}} P_{j}
$$

The inequality $D_{i} \leq P_{i}$ shows that $D_{i}$ commutes with each $P_{j}$, so $D_{i} \in \mathfrak{M}_{0}^{\prime}$ for all $i$, thus $\sum_{i=1}^{\infty}\left(1 / 2^{i}\right) D_{i} \in \mathfrak{M}_{0}^{\prime}$. But $\sum_{i=1}^{\infty}\left(1 / 2^{i}\right) D_{i}$ is the density matrix of the state $\omega$, and the last relation shows that it is also the density matrix of $\omega_{1}=\omega \mid \mathfrak{M}_{0}^{\prime}$. Calculating $D_{j, 0} D_{\omega_{1}}$ we get

$$
D_{j, 0} D_{\omega_{1}}=\frac{1}{d_{j}} P_{j} \sum_{i=1}^{\infty} \frac{1}{2^{i}} D_{i}=\frac{1}{2^{j} d_{j}} D_{j} .
$$

Now the factorization theorem, [1, Theorem 4], states that there is $z \in \mathfrak{M}_{0} \cap \mathfrak{M}_{0}^{\prime}=\mathfrak{M}_{0}$ such that

$$
D_{j}=D_{j, 0} D_{\omega_{1}} z, \quad \text { for all } j,
$$

which means that

$$
D_{j}=\frac{1}{2^{j} d_{j}} D_{j} z \quad \text { for all } j
$$

This is possible only for

$$
z=\sum_{i=1}^{\infty} 2^{i} d_{i} P_{i}
$$


but then

$$
2^{i} d_{i} \geq 2^{i} \rightarrow \infty
$$

thus $z$ is unbounded. This contradiction shows that the factorization theorem in its present form fails.

What we have seen above is not the only trouble with the factorization theorem. Namely, it employs the density matrices $D_{j, 0}, D_{\omega_{1}}$ of the states $\rho_{j} \mid \mathfrak{M}_{0}$ and $\omega_{1}=\omega \mid \mathfrak{M}_{0}^{\prime}$, respectively. But in order that such density matrices exist the canonical trace tr on $\mathfrak{M}=\mathbb{B}(\mathcal{H})$ restricted to the algebras $\mathfrak{M}_{0}$ and $\mathfrak{M}_{0}^{\prime}$ must be semifinite. The following simple example shows that this is not guaranteed by the assumptions of the factorization theorem.

Example 3 We copy the setup of Example 2 with the only difference that now the projections $P_{i}$ are infinite dimensional and the eigenvalues of the density matrices $D_{i}$ are all the same and equal $\frac{1}{2^{k}}, k=1,2, \ldots$ The entropies of $\rho_{i}$ are

$$
S\left(\rho_{i}\right)=-\sum_{k=1}^{\infty} \frac{1}{2^{k}} \log \frac{1}{2^{k}}=2,
$$

and for the entropy of $\omega$ we get

$$
S(\omega)=-\sum_{i=1}^{\infty} \sum_{k=1}^{\infty} \frac{1}{2^{i}} \frac{1}{2^{k}} \log \frac{1}{2^{i}} \frac{1}{2^{k}}=4 .
$$

Defining $\mathfrak{M}_{0}$ and $\mathbb{E}$ by formulas (3) and (4), respectively, we again obtain that $\mathbb{E}$ is a normal conditional expectation such that the states $\rho_{i}$ are $\mathbb{E}$-invariant, so $\mathfrak{M}_{0}$ is sufficient for $\left\{\rho_{i}\right.$ : $i=1,2, \ldots\}$ in Umegaki's sense, and that $\mathfrak{M}_{0}$ is invariant with respect to the modular automorphism group $\left\{\sigma_{t}^{\omega}\right\}$. However, the factorization theorem does not hold because there are no density matrices of the states $\rho_{j} \mid \mathfrak{M}_{0}$.

Now, a careful inspection of the considerations before the factorization theorem ([1, p. 269]) shows that its conclusion holds in a slightly weaker sense. Namely, a unitary group $\left\{z_{t}\right\}$ in the center of the algebra $\mathfrak{M}_{0}^{\prime} \cap \mathfrak{M}$ is defined (line 11 from bottom of page) and it is concluded that $z_{t}=z^{i t}$ for some positive element $z$ in the center. But this is wrong, because the generator of a unitary group in a von Neumann algebra needn't belong to this algebra (it may be unbounded); instead, it is affiliated with the algebra. (This is exactly the situation in our Example 2 where $z$ is affiliated with $\mathfrak{M}_{0}$.) Thus the basic formula of the factorization theorem would read

$$
D_{\theta}=\overline{D_{\theta, 0} D_{\omega_{1}} z} \quad(\text { cf. formula (12) in [1]), }
$$

for some positive operator $z$ affiliated with the center of $\mathfrak{M}_{0}^{\prime} \cap \mathfrak{M}$, but still under the assumption that the densities $D_{\theta}, D_{\theta, 0}$ and $D_{\omega_{1}}$ of $\phi_{\theta}, \phi_{\theta} \mid \mathfrak{M}_{0}$ and $\omega \mid \mathfrak{M}_{0}^{\prime} \cap \mathfrak{M}$, respectively, exist.

We show elsewhere that for finite von Neumann algebras the factorization theorem with formula (5) holds with an appropriately defined notion of density matrix which guarantees its existence. 


\section{Completeness, Ancillarity and Quantum Basu's Theorem}

Analogously to the classical case we can define completeness of a quantum statistic, or more generally, of a von Neumann algebra.

A von Neumann algebra $\mathfrak{M}$ is said to be complete with respect to a family of states $\left\{\rho_{\theta}: \theta \in \Theta\right\}$ if for each $A \in \mathfrak{M}$ from the equality $\rho_{\theta}(A)=0$ for all $\theta \in \Theta$ it follows that $A=0$.

It is immediately seen that the completeness of the algebra $\mathfrak{M}$ with respect to $\left\{\rho_{\theta}: \theta \in \Theta\right\}$ is equivalent to separating the points of this algebra by $\left\{\rho_{\theta}: \theta \in \Theta\right\}$.

It turns out that for complete subalgebras sufficiency is equivalent to sufficiency in Umegaki's (and thus Petz's) sense.

Theorem 3 Let $\left\{\rho_{\theta}: \theta \in \Theta\right\}$ be a family of normal states on a von Neumann algebra $\mathfrak{M}$, and let $\mathfrak{N}$ be a von Neumann subalgebra of $\mathfrak{M}$ sufficient and complete with respect to this family. Then $\mathfrak{N}$ is sufficient in Umegaki's sense.

Proof Let $\alpha: \mathfrak{M} \rightarrow \mathfrak{N}$ be a map defining the sufficiency of $\mathfrak{N}$. For an arbitrary $T \in \mathfrak{N}$ and arbitrary $\theta \in \Theta$ we have

$$
\rho_{\theta}(\alpha(T))=\rho_{\theta}(T),
$$

which on account of the completeness of $\mathfrak{N}$ gives the equality

$$
\alpha(T)=T \text {. }
$$

Thus $\alpha$ is a positive projection of norm one from $\mathfrak{M}$ onto $\mathfrak{N}$, i.e. a conditional expectation.

The next theorem shows a connection between complete and minimal algebras.

Theorem 4 Let $\left\{\rho_{\theta}: \theta \in \Theta\right\}$ be a faithful family of normal states on a von Neumann algebra $\mathfrak{M}$, and let $\mathfrak{N}$ be a von Neumann subalgebra of $\mathfrak{M}$ sufficient and complete with respect to this family. Then $\mathfrak{N}$ is minimal.

Proof Let $\mathfrak{M}_{\min }$ be the minimal sufficient subalgebra and let $\alpha: \mathfrak{M} \rightarrow \mathfrak{M}_{\min }$ be a map defining this sufficiency. The algebra $\mathfrak{N}$ is, according to Theorem 3 , sufficient in Umegaki's sense; let $\mathbb{E}: \mathfrak{M} \rightarrow \mathfrak{N}$ be a conditional expectation with respect to which the states $\rho_{\theta}$ are invariant. For each $A \in \mathfrak{M}$ and each $\theta \in \Theta$ we have

$$
\rho_{\theta}(\mathbb{E}(\alpha(A)))=\rho_{\theta}(\alpha(A))=\rho_{\theta}(A)
$$

and since $\mathbb{E}(\alpha(A)) \in \mathfrak{N}$, and $\alpha(A) \in \mathfrak{M}_{\min } \subset \mathfrak{N}$ by the minimality of $\mathfrak{M}_{\text {min }}$, the completeness of $\mathfrak{N}$ yields

$$
\mathbb{E}(\alpha(A))=\alpha(A)=A \quad \text { for each } A \in \mathfrak{N}
$$

thus for each $A \in \mathfrak{N}$ we have $A=\alpha(A) \in \mathfrak{M}_{\min }$, which shows that $\mathfrak{N} \subset \mathfrak{M}_{\min }$, so $\mathfrak{N}=\mathfrak{M}_{\min }$, consequently, $\mathfrak{N}$ is minimal.

Remark 2 The assumption of the faithfulness of the family $\left\{\rho_{\theta}: \theta \in \Theta\right\}$ was exploited only for obtaining the existence of the minimal sufficient algebra. It is easily seen that the proof of the above theorem without this assumption remains the same if this existence is taken for granted. 
Our final aim is a quantum counterpart of the classical Basu theorem. For this we need the notions of ancillarity and independence which are again fully analogous to those in the classical case.

A subalgebra $\mathfrak{N}$ of $\mathfrak{M}$ is said to be ancillary with respect to the family of states $\left\{\rho_{\theta}: \theta \in \Theta\right\}$ if $\rho_{\theta} \mid \mathfrak{N}$ is the same for all $\theta \in \Theta$.

Let $\rho$ be a state on a von Neumann algebra $\mathfrak{M}$, and let $\mathfrak{N}$ and $\mathfrak{R}$ be two von Neumann subalgebras of the algebra $\mathfrak{M}$. The algebras $\mathfrak{N}$ and $\mathfrak{R}$ are said to be independent in the state $\rho$ if for any $T \in \mathfrak{N}, R \in \mathfrak{R}$ we have

$$
\rho(T R)=\rho(T) \rho(R) .
$$

We have the following quantum Basu's theorem.

Theorem 5 Let $\left\{\rho_{\theta}: \theta \in \Theta\right\}$ be a family of normal states on a von Neumann algebra $\mathfrak{M}$, and let $\mathfrak{N}$ and $\mathfrak{R}$ be von Neumann subalgebras of $\mathfrak{M}$.

(i) If $\mathfrak{N}$ is ancillary, and $\mathfrak{R}$ is sufficient and complete with respect to $\left\{\rho_{\theta}\right\}$, then $\mathfrak{N}$ and $\mathfrak{R}$ are independent in each state $\rho_{\theta}$.

(ii) If the family $\left\{\rho_{\theta}\right\}$ is faithful and such that no two states in it have mutually orthogonal supports, $\mathfrak{R}$ is sufficient with respect to $\left\{\rho_{\theta}\right\}$, and $\mathfrak{N}$ and $\mathfrak{R}$ are independent in each state $\rho_{\theta}$, then $\mathfrak{N}$ is ancillary with respect to $\left\{\rho_{\theta}\right\}$.

Proof (i) Set $\rho_{\theta} \mid \mathfrak{N}=\omega$. Since $\mathfrak{R}$ is sufficient and complete we infer on account of Theorem 3 that it is sufficient in Umegaki's sense. Let $\mathbb{E}: \mathfrak{M} \rightarrow \mathfrak{R}$ be a conditional expectation onto $\mathfrak{R}$ such that the states $\rho_{\theta}$ are $\mathbb{E}$-invariant. For arbitrary $\theta \in \Theta$ and arbitrary $T \in \mathfrak{N}$ we obtain the equality

$$
\rho_{\theta}(\mathbb{E}(T)-\omega(T) \mathbb{1})=\rho_{\theta}(T)-\omega(T)=0
$$

and the completeness of $\Re$ gives

$$
\mathbb{E}(T)=\omega(T) \mathbb{1} .
$$

For arbitrary $R \in \mathfrak{R}, T \in \mathfrak{N}$ and $\theta \in \Theta$ the equality above, the $\mathbb{E}$-invariance of the states $\rho_{\theta}$, and properties of a conditional expectation yield

$$
\begin{aligned}
\rho_{\theta}(R T) & =\rho_{\theta}(\mathbb{E}(R T))=\rho_{\theta}(R \mathbb{E}(T))=\rho_{\theta}(\omega(T) R) \\
& =\rho_{\theta}(R) \omega(T)=\rho_{\theta}(R) \rho_{\theta}(T),
\end{aligned}
$$

which proves the independence of the algebras $\mathfrak{N}$ and $\mathfrak{R}$ in the state $\rho_{\theta}$.

(ii) We shall employ the setup and notation of Theorem 1 . We have $\mathfrak{A} \subset \mathfrak{M}_{\min } \subset \mathfrak{R}$, and let $\mathbb{E}: \mathfrak{M} \rightarrow \mathfrak{A}$ be the projection as in the proof of Theorem 1 . For any $\theta \in \Theta, R \in \mathfrak{A}$, and $T \in \mathfrak{N}$, we have on account of the relation $\rho_{\theta}(R T)=\rho_{\theta}(R) \rho_{\theta}(T)=\rho_{\theta}(T R)$, and properties of $\mathbb{E}$, the equality

$$
\rho_{\theta}(R) \rho_{\theta}(T)=\rho_{\theta}(R \circ T)=\rho_{\theta}(\mathbb{E}(R \circ T))=\rho_{\theta}(R \circ \mathbb{E} T),
$$

yielding

$$
\rho_{\theta}\left(R \circ\left(\mathbb{E} T-\rho_{\theta}(T) \mathbb{1}\right)\right)=0 .
$$


Taking $R=\left(\mathbb{E} T-\rho_{\theta}(T) \mathbb{1}\right)^{*}$ we obtain

$$
\rho_{\theta}\left(\left(\mathbb{E} T-\rho_{\theta}(T) \mathbb{1}\right)^{*}\left(\mathbb{E} T-\rho_{\theta}(T) \mathbb{1}\right)\right)=0,
$$

which gives the equality

$$
\left(\mathbb{E} T-\rho_{\theta}(T) \mathbb{1}\right) \mathrm{s}\left(\rho_{\theta}\right)=0,
$$

i.e.

$$
(\mathbb{E} T) \mathrm{s}\left(\rho_{\theta}\right)=\rho_{\theta}(T) \mathrm{s}\left(\rho_{\theta}\right) .
$$

In particular, it follows that $\mathbb{E} T$ commutes with all supports $\mathrm{s}\left(\rho_{\theta}\right)$, so for arbitrary $\theta_{1}, \theta_{2} \in \Theta$ we get from (6)

$$
\begin{aligned}
\rho_{\theta_{1}}(T) \mathrm{s}\left(\rho_{\theta_{1}}\right) \mathrm{s}\left(\rho_{\theta_{2}}\right) & =(\mathbb{E} T) \mathrm{s}\left(\rho_{\theta_{1}}\right) \mathrm{s}\left(\rho_{\theta_{2}}\right)=\mathrm{s}\left(\rho_{\theta_{1}}\right)(\mathbb{E} T) \mathrm{s}\left(\rho_{\theta_{2}}\right) \\
& =\rho_{\theta_{2}}(T) \mathrm{s}\left(\rho_{\theta_{1}}\right) \mathrm{s}\left(\rho_{\theta_{2}}\right) .
\end{aligned}
$$

Since by assumption $\mathrm{s}\left(\rho_{\theta_{1}}\right) \mathrm{s}\left(\rho_{\theta_{2}}\right) \neq 0$, we obtain $\rho_{\theta_{1}}(T)=\rho_{\theta_{2}}(T)$ showing the claim.

Open Access This article is distributed under the terms of the Creative Commons Attribution License which permits any use, distribution, and reproduction in any medium, provided the original author(s) and the source are credited.

\section{References}

1. Jenčová, A., Petz, D.: Sufficiency in quantum statistical inference. Commun. Math. Phys. 263, 259-276 (2006)

2. Jenčová, A., Petz, D.: Sufficiency in quantum statistical inference. A survey with examples. Infin. Dimens. Anal. Quantum Probab. Relat. Top. 9, 331-352 (2006)

3. Kümmerer, B., Nagel, R.: Mean ergodic semigroups on $W^{*}$-algebras. Acta Sci. Math. 41, 151-159 (1979)

4. Ohya, M., Petz, D.: Quantum Theory and Its Use. Springer, Berlin (1993)

5. Petz, D.: Sufficient subalgebras and the relative entropy of states of a von Neumann algebra. Commun. Math. Phys. 105, 123-131 (1986)

6. Petz, D.: Sufficiency of channels over von Neumann algebras. Q. J. Math. 39, 907-1008 (1988)

7. Thomsen, K.E.: Invariant states for positive operator semigroups. Stud. Math. 81, 285-291 (1985)

8. Umegaki, H.: Conditional expectation in an operator algebra, III. Kodai Math. Semin. Rep. 11, 51-64 (1959)

9. Umegaki, H.: Conditional expectation in an operator algebra, IV (entropy and information). Kodai Math. Semin. Rep. 14, 59-85 (1962) 